\title{
Rationalisierung, Rationierung, Priorisierung: Terminologie und ethische Begründungsansätze zur Allokation bei begrenzten Ressourcen in der Hämatologie/Onkologie
}

\author{
Eva Winkler \\ Medizinische Klinik und Poliklinik III, Ludwig-Maximilian-Universität, Campus Großhadern, München, Germany
}

\section{Schlüsselwörter}

Rationierung · Rationalisierung · Priorisierung ·

Verteilungsgerechtigkeit · Ethische Verteilungskriterien

\begin{abstract}
Zusammenfassung
Die Onkologie trägt mit ihren zahlreichen teuren Neueinführungen nicht unerheblich dazu bei, dass der medizinische Fortschritt teuer ist. Zudem nimmt durch den demographischen Wandel und die steigende Lebenserwartung die Zahl der Krebspatienten zu, die von diesem Fortschritt profitieren möchten. Da auch im Gesundheitswesen die Mittel begrenzt sind, stellt sich die Frage nach der gerechten Verteilung der vorhandenen Ressourcen mit immer größerer Dringlichkeit. Da der Umgang mit Knappheit ein typisches gerechtigkeitstheoretisches Problem ist, diskutiert dieser Artikel zunächst verschiedene Strategien im Umgang mit knapper werdenden Ressourcen (Rationalisierung, Rationierung und Priorisierung). Es werden dann prozedurale und inhaltliche Kriterien vorgestellt, die dabei helfen, nutzbringende Gesundheitsleistungen gerecht zu verteilen. Es gibt verschiedene Strategien, den Verbrauch knapper werdender Mittel zu senken: Mittels Rationalisierung werden Effizienzreserven ausgeschöpft, mittels Rationierung werden Gesundheitsleistungen begrenzt und damit auch nutzbringende Maßnahmen aus dem Leistungskatalog ausgeschlossen. Rationierung kann implizit, also verdeckt erfolgen, z.B. durch Budgetierung oder die Einführung von Wartezeiten, oder explizit durch transparente Festlegung der Leistungen, auf die verzichtet werden soll. Hierfür ist häufig eine Ordnung medizinischer Leistungen nach ihrer Wichtigkeit die Voraussetzung (Priorisierung). Für die Akzeptanz und die Güte einer Entscheidung zum Verzicht auf nutzbringende Leistungen sind aus gerechtigkeitstheoretischer Sicht sowohl prozedurale Kriterien als auch inhaltliche Verteilungsprinzipien (Gleichheit, Dringlichkeit, Nutzen) relevant.
\end{abstract}

\author{
Keywords \\ Rationalization · Rationing · Prioritization . \\ Distributive justice $\cdot$ Ethical allocation criteria
}

\section{Summary}

Rationalization, Rationing, Prioritization: Terminology and Ethical Approaches to the Allocation of Limited Resources in Hematology/Oncology

The field of oncology with its numerous high-priced innovations contributes considerably to the fact that medical progress is expensive. Additionally, due to the demographic changes and the increasing life expectancy, a growing number of cancer patients want to profit from this progress. Since resources are limited also in the health system, the fair distribution of the available resources urgently needs to be addressed. Dealing with scarcity is a typical problem in the domain of justice theory; therefore, this article first discusses different strategies to manage limited resources: rationalization, rationing, and prioritization. It then presents substantive as well as procedural criteria that assist in the just distribution of effective health benefits. There are various strategies to reduce the utilization of limited resources: Rationalization means that efficiency reserves are being exhausted; by means of rationing, effective health benefits are withheld due to cost considerations. Rationing can occur implicitly and thus covertly, e.g. through budgeting or the implementation of waiting periods, or explicitly, through transparent rules or policies about healthcare coverage. Ranking medical treatments according to their importance (prioritization) is often a prerequisite for rationing decisions. In terms of requirements of justice, both procedural and substantive criteria (e.g. equality, urgency, benefit) are relevant for the acceptance and quality of a decision to limit access to effective health benefits.

\section{KARGER \\ Fax +497614520714 \\ Information@Karger.de}

www.karger.com
() 2011 S. Karger GmbH, Freiburg

0378-584X/11/3413-0002\$38.00/0

Accessible online at:

www.karger.com/onk
Dr. med. Dr. phil. Eva Winkle

Medizinische Klinik und Poliklinik III

Ludwig-Maximilian-Universität, Campus Großhadern

Marchioninistraße 15, 81377 München, Germany

Tel. +49 89 7095-2551, Fax -5550

eva.winkler@med.uni-muenchen.de 


\section{Hintergrund}

Der medizinische Fortschritt mit teuren neuen Therapieverfahren und der demographische Wandel sind gemeinhin als Ursache für die steigenden Kosten unseres Gesundheitswesens bekannt. Das Ausmaß der Veränderungen in der Onkologie wird anhand der Zahlen zur Prävalenzentwicklung von Krebserkrankungen der letzten 30 Jahre, die das RobertKoch-Institut Anfang 2010 vorgelegt hat, besonders deutlich: Von 1990 bis 2004 ist die Prävalenz von Krebserkrankungen insgesamt um etwa $35 \%$ bei den Frauen und $80 \%$ bei den Männern angestiegen. Der Anstieg wird zum einen auf die Zunahme des Anteils älterer Menschen zurückgeführt, die höhere Krebserkrankungsraten haben (der deutlichste Anstieg war bei den 60-79-jährigen Männern zu verzeichnen), zum anderen aber auch auf den medizinischen Fortschritt mit einer Verbesserung der 5-Jahres-Überlebensraten von 38 auf 53\% für Männer und von 50 auf $60 \%$ bei Frauen mit Krebserkrankungen, von Anfang der 1980erJahre bis 2004 [1]. Das zeigt, dass in relativ kurzer Zeit die Zahl der an Krebs erkrankten Patientinnen und Patienten und auch die Dauer der Behandlung erheblich gestiegen sind. Hinzu kommt, dass die Jahrestherapiekosten im gleichen Zeitraum gerade durch neue zielgerichtete Therapeutika, zum Beispiel in der Darmkrebsbehandlung, um das 450-Fache gestiegen sind [2]. Diese Entwicklungen schlagen sich auch in den Krankheitskosten für bösartige Neubildungen in Deutschland nieder, die innerhalb von nur 4 Jahren (2002-2006) um ein Drittel gestiegen sind, während der Anstieg der gesamten Krankheitskosten im gleichen Zeitraum nur etwa $8 \%$ betrug [3]. Diese Situation wird sich noch weiter zuspitzen, wenn der Anteil der über 65-Jährigen an der Gesamtbevölkerung wie prognostiziert von jetzt 15\% auf zirka 25\% im Jahr 2040 steigen wird und gleichzeitig weitere neue Wirkstoffe mit Jahrestherapiekosten zwischen 40000 und 100000 Euro zugelassen werden. Vor diesem Hintergrund wird deutlich, dass die Entwicklungen in der Onkologie ganz wesentlich zur Leistungs- und Kostensteigerung im Gesundheitswesen beitragen und die Fachvertreter daher in besonderer Weise gefragt sind, ihren Umgang mit knappen Ressourcen zu rechtfertigen. Die bereits jetzt abzusehende Kostenentwicklung macht deutlich, dass dieser nicht mit einer Umverteilung der verfügbaren finanziellen Mittel zugunsten des Gesundheitswesens zu begegnen ist: Zum einen würde damit nur ein kurzer zeitlicher Aufschub erkauft, bis sich die Frage nach dem Umgang mit begrenzten Mitteln erneut stellt, und zum anderen müssten diese Mittel in anderen sozialstaatlichen Bereichen wie Bildung, Umweltschutz oder Arbeitslosenunterstützung abgezogen werden.

Gesundheit ist zwar ein hohes Gut, für das kaum Kosten gescheut werden. Da sich jedoch das Problem der Mittelknappheit nicht durch das Erreichen eines Sättigungsgrades entschärfen lässt, muss eine Gesellschaft letztlich Strategien zum Umgang mit begrenzten Ressourcen entwickeln.

\section{Rationalisierung, Preisregulierung und Rationierung als Strategien im Umgang mit begrenzten Ressourcen}

Als primäre Antwort auf Situationen, in denen absehbar ist, dass die Mittel knapp werden und nicht alles Nutzbringende finanzierbar ist, ist es ethisch wie ökonomisch geboten, die Effizienzreserven des Systems auszuschöpfen - also zu rationalisieren. Rationalisierungspotenziale sieht etwa der Sachverständigenrat zur Begutachtung der Entwicklung im Gesundheitswesen vor allem in einer besseren Vernetzung der ambulanten und stationären Versorgung und in einer besseren Kooperation der Gesundheitsberufe [4]. Voraussetzung für eine Effizienzsteigerung ist darüber hinaus jedoch das Wissen, wie das Therapieziel möglichst kostengünstig erreicht werden kann. Hierbei helfen Verfahren zur systematischen, evidenzbasierten Nutzenbewertung wie Metaanalysen, S3Leitlinien, Bewertungen des Instituts für Qualität und Wirtschaftlichkeit (IQWiG) (siehe auch Beitrag von W.-D. Ludwig und J. Schildmann in diesem Supplement). Eine weitere Möglichkeit, Kosten zu sparen, besteht darin, die Preise z.B. für Arzneimittel direkt zu regulieren und Höchstbeträge für Arzneimittel festzusetzen. Die Basis für die Festsetzungen erarbeitet gegenwärtig das IQWiG durch die Entwicklung einer Methode zur Bestimmung von Effizienzgrenzen [5]. Noch direkter greift das von Gesundheitsminister Philipp Rösler ausgearbeitete Arzneimittel-Sparpaket in die Kostenentwicklung ein, da es seit August 2010 den Herstellerrabatt an die Kassen von $6 \%$ auf $16 \%$ erhöht hat.

Da das Einsparpotenzial des Systems von vielen als nicht ausreichend eingeschätzt wird [6, 7], um den eingangs aufgeführten Entwicklungen Rechnung zu tragen, bleibt als Alternative im Umgang mit der Mittelknappheit nur die Rationierung. Rationieren heißt, auf nutzbringende medizinische Maßnahmen zu verzichten, d.h. auf Maßnahmen, die den Gesundheitszustand der Betroffenen verbessern oder eine Verschlechterung verhindern oder verzögern können. Wenn Rationierung unumgänglich ist, wird man die Maßnahmen zunächst nach Wichtigkeit ordnen, um dann zunächst auf die Maßnahmen zu verzichten, die als wenig wichtig eingestuft wurden. Diesen Vorgang nennt man Priorisierung. Er ist der Rationierungsentscheidung vorgeschaltet.

\section{Allokationsebenen und Rationierungsmechanismen}

Rationierungsentscheidungen, die letztlich auch die Versorgung der Menschen mit onkologischen Erkrankungen bestimmen, werden nach einem verbreiteten Modell 4 Allokationsebenen zugeordnet [8]. Auf der Makroebene I wird festgelegt, welcher Anteil des Bruttosozialprodukts für das Gesundheitssystem zur Verfügung steht und wie viel in andere staatliche Ziele wie Bildung oder Sicherheit investiert wird. Auf der Makroebene II werden die Budgets entsprechend der verschiedenen Ziele des Gesundheitswesens aufgeteilt, z.B. auf Prävention, Therapie, 
Rehabilitation oder medizinische Forschung. Auf der mittleren Ebene (auch als Mesoebene oder Mikroebene I bezeichnet), sollten die «Prinzipien festgelegt werden, nach denen Ressourcen der Gesundheitsversorgung an bestimmte Einzelpersonen zugeteilt werden» ([8]: S. 42). Hier werden Gesundheitsprogramme entwickelt, die für bestimmte Leistungsempfänger gedacht sind, und festgelegt, wer in die Gruppe der Leistungsempfänger hineinfällt bzw. nicht hineinfällt (z.B. für ein bestimmtes Medikament). Fehlen objektive Kriterien der Mittelverteilung auf dieser Ebene, findet die Rationierung unweigerlich auf der untersten Ebene (Mikroebene II) der Behandlungsentscheidung von Arzt und Patient statt. Rationierung auf dieser Ebene ist häufig nicht transparent und folgt keinen festgelegten Kriterien. Rationierung wird als «hart» bezeichnet, wenn es nicht möglich ist, neben dem staatlich festgelegten Leistungsniveau Gesundheitsleistungen hinzuzukaufen. Damit wird zwar Versorgungsgleichheit angestrebt, aber die Freiheit derer, die privat finanziert Zusatzleistungen in Anspruch nehmen wollen, so beschnitten, dass diese Form der Rationierung in liberalen Gesellschaften nicht praktiziert wird. Es wird kontrovers diskutiert, welche Rolle Ärzte bei der Rationierung am Krankenbett spielen sollen [9-11]. Einerseits ist die Basis für den Behandlungsvertrag und das Vertrauensverhältnis zwischen Arzt und Patient eine auf das Patientenwohl ausgerichtete und nicht primär am Gemeinwohl orientierte Behandlung. Andererseits macht der Sozialgesetzgeber die Rücksicht auf die Bedürfnisse der Allgemeinheit durch die Notwendigkeits- und Wirtschaftlichkeitsprüfung zur Pflicht des Arztes als Leistungserbringer: $§ 12$ Abs. $1 \mathrm{~S}$. 2 SGB V: «Leistungen, die nicht notwendig oder wirtschaftlich sind, können Versicherte nicht beanspruchen, dürfen die Leistungserbringer nicht bewirken und die Krankenkassen nicht bewilligen.» Ein Argument hierfür ist, dass der behandelnde Arzt zumindest die Notwendigkeit der Behandlung im Einzelfall am besten beurteilen und so dem Einzelfall besser gerecht werden kann. Dieser Rollenkonflikt, dem Patientenwohl und der Versicherungsgemeinschaft verpflichtet $\mathrm{zu}$ sein, wird von vielen Medizinern heute als belastend wahrgenommen und ist häufig schwer aufzulösen (siehe auch die Beiträge von D. Strech und J. Schildmann et al. in diesem Supplement). Wenn Rationierungsentscheidungen allein auf der Arzt-Patienten-Ebene getroffen werden, birgt dies zudem die Gefahr, dass gleiche Fälle abhängig von den individuellen Bewertungsgrenzen unterschiedlicher Ärzte und den Üblichkeiten in ihrem Fachgebiet nicht gleich behandelt werden. Das würde gegen den Gleichheitsgrundsatz und damit gegen ein grundsätzliches Gerechtigkeitsprinzip verstoßen. Umfragestudien zeigen, dass sich Ärzte aufgrund des Kostendrucks auch heute schon mit Priorisierungen und Rationierungen konfrontiert sehen $[12,13]$. Die Entscheidung, ob Therapien mit grenzwertigem Lebenszeit- oder Lebensqualitätsgewinn angeboten und finanziert werden, sollte daher oberhalb der Arzt-Patienten-Beziehung nach transparenten und expliziten Kriterien getroffen werden (explizite Rationierung). Hierfür ist jedoch eine gesellschaftliche Einigung auf allgemein anerkannte explizite Rationierungskriterien nötig, die politisch sehr viel schwieriger zu erreichen ist als die implizite Rationierung durch finanzielle Steuerungssysteme wie Budgets, Diagnosis-Related Group (DRG)-Pauschalen oder Kopfpauschalen (siehe auch den Beitrag von M. Thalheimer in diesem Supplement). Aus medizinethischer Perspektive ist die implizite Rationierung vor allem wegen der fehlenden Transparenz hinsichtlich der Kriterien zur Verteilung knapper Mittel und der daraus resultierenden Gefahr der Ungleichbehandlung fragwürdig.

\section{Was ist gerecht? Rationierungskriterien bei der Verteilung von Gesundheitsleistungen}

Die Frage, welche Verteilung von nutzbringenden Gesundheitsleistungen unter Knappheitsbedingungen gerecht ist, lässt sich nicht konsensfähig beantworten, weil es verschiedene Auffassungen darüber gibt, was Gerechtigkeit von uns fordert: Gleichbehandlung oder Vorrang für die in Not oder für die, die am meisten von einer Behandlung profitieren? Während egalitäre Gerechtigkeitstheorien das Gleichheitsgebot in den Mittelpunkt stellen, verwenden utilitaristische Rationierungsstrategien die Nutzenmaximierung als wichtigstes Verteilungskriterium. Das Gleichheitsprinzip fordert für gleich Bedürftige die gleiche Behandlung, wobei es sich im Falle unteilbarer Güter wie in der Transplantationsmedizin um gleiche Ansprüche auf ein Organ bei gleicher Bedürftigkeit handelt [14, 15]. Dabei wird deutlich, dass allein auf Grundlage der Chancengleichheit keine Entscheidung herbeigeführt wird, auf welche Maßnahme verzichtet werden soll, sondern vor allem eine ungleiche Verteilung der Maßnahmen verhindert wird. Gerade am Beispiel der Organvergabe wird deutlich, dass sich bei gleicher Bedürftigkeit ein deutlicher Unterschied in der Nutzenwahrscheinlichkeit, Nutzendauer oder Nutzenqualität als zusätzliches Entscheidungskriterium aufdrängt. Diese Kriterien folgen der utilitaristischen Logik, der es um die Maximierung des gesamtgesellschaftlichen Nutzens durch die Ausgaben im Gesundheitssystem geht. Das am häufigsten verwendete Nutzenmaß sind die «qualitätsbereinigten Lebensjahre» (QALYs) [16]. Diese machen medizinische Maßnahmen hinsichtlich des durch sie ermöglichten Zugewinns an Lebensjahren und -qualität vergleichbar. Daraus resultiert jedoch eine systematische Benachteiligung von Patienten im fortgeschrittenen Lebensalter sowie von Menschen mit seltenen oder unheilbaren Erkrankungen. Diese stellen gerade im Fachgebiet der Hämatologie und Onkologie eine relevante Patientengruppe dar. Auch wenn das Recht auf gleiche Gesundheitsversorgung eine klare Priorität der Gleichheit vor Effizienzaspekten nahelegt, wird aus den oben dargestellten Kostenentwicklungen deutlich, dass auch die Frage nach dem finanzierbaren Grenznutzen gestellt werden muss, damit nicht überproportional große Anteile der knappen Mittel für einen marginalen Nutzen einiger Weniger verbraucht werden. Wünschenswert wäre ein gesellschaftlich konsensfähiger Schwellenwert, unter dem eine Maßnahme für zu wenig wirksam angesehen wird, als dass sie 
durch das öffentlich finanzierte Gesundheitswesen abgedeckt werden soll. Solange es einen solchen Konsens noch nicht gibt, ist es denkbar, dass medizinische Fachgesellschaften, Krankenversicherer oder auch Krankenhäuser Empfehlungen zum kostenbewussten Einsatz von Therapien mit geringer Wirksamkeit erarbeiten. Denn wenn Rationierung unvermeidbar ist, dann sollten zunächst die Therapien mit marginalem medizinischem Nutzen zur Disposition gestellt werden, da hier bei vertretbarem Gewinnverzicht Kosten eingespart werden können [17]. Dieses Ordnen medizinischer Leistungen nach ihrer Wichtigkeit wird als Priorisierung bezeichnet. Der OregonHealth-Plan ist zum Beispiel das Ergebnis eines Ende der 1980er-Jahre begonnenen Prozesses, in dessen Rahmen die Oregon Health Services Commission auf der Grundlage öffentlicher Anhörungen, Kosten-Nutzen-Bewertungen und Umfragen eine Priorisierung von Diagnosen und Behandlungsmaßnahmen vorgenommen hat, die aus öffentlichen Geldern finanziert werden sollten [18]. Wie bei der Rationierung stellt sich auch bei der Priorisierung die Frage nach den Kriterien, die eine Listung als «wichtig» und «weniger wichtig» rechtfertigen. Da es nicht ein führendes Gerechtigkeitskriterium gibt, ist die Zusammenführung der relevanten Rationierungskriterien - wie etwa Gleichheit, Dringlichkeit, Nutzen in ein konsistentes Bewertungsverfahren wünschenswert. Der Prozess der Entscheidungsfindung und das Verteilungsverfahren gewinnen damit zunehmend an Bedeutung. Daher sollten prozedurale Gerechtigkeitskriterien ebenfalls berücksichtigt werden: Der Entscheidungsprozess sollte transparent und rational nachvollziehbar sein und alle von der Entscheidung Betroffenen (Stakeholder) einbeziehen.

\section{Fazit}

Da Einsparpotenziale gemeinhin als nicht ausreichend beurteilt werden, um den steigenden Kosten im Gesundheitswesen zu begegnen, scheint der Verzicht auf nutzbringende Leistungen (Rationierung) unumgehbar. Aus ethischer Sicht ist es wünschenswert, explizite Rationierungskriterien oberhalb der Ebene des Arzt-Patienten-Verhältnisses festzulegen - also auf der Planungsebene des Gesundheitsministeriums, des Krankenversicherers, des Krankenhauses oder unter Berufung auf Empfehlungen der Fachgesellschaften. Damit wäre der Gleichbehandlung der Patienten gedient und der Arzt in seiner Doppelrolle als Patientenfürsprecher und Wirtschaftlichkeitsprüfer entlastet. Andererseits schränken diese Vorgaben den Entscheidungsspielraum des Arztes und damit seine Möglichkeiten ein, dem einzelnen Patienten in seiner Krankheitssituation gerecht zu werden. Zudem ist eine politische Verständigung auf die Prioritäten im Gesundheitswesen langwieriger in der Umsetzung als das Arbeiten mit Budgets oder anderen impliziten Rationierungsmechanismen. Bei der Priorisierung von medizinischen Maßnahmen sollten verschiedene gesundheitsrelevante Werte - wie Nutzen, Chancengleichheit, Dringlichkeit - berücksichtigt werden.

\section{Disclosure Statement}

Die Autorin gibt an, dass kein Interessenkonflikt besteht.

\section{Referenzen}

1 Robert Koch Institut: Verbreitung von Krebserkrankungen in Deutschland. Entwicklung der Prävalenzen zwischen 1990 und 2010. www.rki.de/ cln_178/nn_204124/sid_677B981E96C280FCDA12 616F4195688E/DE/Content/GBE/DachdokKrebs/ Praevalenz/Lokalisationen/Krebslokalisationen_ tabelle_gesamt.html, letzter Zugriff: 05.11.2010.

2 Bach PB: Limits on Medicare's ability to control rising spending on cancer drugs. N Engl J Med 2009;360:626-633.

3 Gesundheitsberichterstattung des Bundes. www. gbe-bund.de, letzter Zugriff: 02.11.2010.

4 Sachverständigenrat zur Begutachtung der Entwicklung im Gesundheitswesen: Kooperation und Verantwortung. Voraussetzungen einer zielorientierten Gesundheitsversorgung (Gutachten 2007). Kurzfassung: www.svr-gesundheit.de.

5 Institut für Qualität und Wirtschaftlichkeit im Gesundheitswesen (IQWiG): Methodik für die Bewertung von Verhältnissen zwischen Nutzen und Kosten im System der deutschen gesetzlichen Krankenversicherung. Version 1,0 (2008). www. iqwig.de/download/08-01-24-Entwurf_Methoden_ Kosten-Nutzen-Bewertung_Version_1_0.pdf.
6 Bundesärztekammer: Stellungnahme der Zentralen Kommission zur Wahrung ethischer Grundsätze in der Medizin und ihren Grenzgebieten (Zentrale Ethikkommission) bei der Bundesärztekammer. Priorisierung medizinischer Leistungen im System der Gesetzlichen Krankenversicherung (GKV). Dtsch Arztebl 2007;104:A2750-A2754

7 Bundesärztekammer: Ulmer Papier. Gesundheitspolitische Leitsätze der Ärzteschaft. Beschluss des 111. Deutschen Ärztetages 2008. www.bundesaerztekammer.de/downloads/UlmerPapierDAET111.pdf.

8 Engelhardt HT: Zielkonflikte in nationalen Gesundheitssystemen; in Sass HM (Hrsg): Ethik und öffentliches Gesundheitswesen. Berlin, Springer, 1988, pp 35-43.

9 Fojo T, Grady C: How much is life worth: cetuximab, non-small cell lung cancer, and the $\$ 440$ billion question. J Natl Cancer Inst 2009;101:10441048.

10 Levinsky NG: The doctor's master. N Engl J Med 1984;311:1573-1575.

11 Ubel PA, Arnold RM: The unbearable rightness of bedside rationing. Physician duties in a climate of cost containment. Arch Intern Med 1995;155:1837_ 1842 .
12 Hurst et al: Physicians' responses to resource constraints. Arch Intern Med 2005;165:639-644.

13 Strech D, Danis M, Löb M, Marckmann G: Ausmaß und Auswirkungen von Rationierung in deutschen Krankenhausern. Ärztliche Einschätzungen aus einer repräsentativen Umfrage. Dtsch Med Wochenschr 2009;134:1261-1266.

14 Gutmann T: Gleichheit vor der Rationierung. Rechtsphilosophische Überlegungen; in Gutmann T, Schmidt VH (Hrsg): Rationierung und Allokation im Gesundheitswesen. Weilerswist, Velbrück Wissenschaft, 2002, p 188.

15 Lübbe W: Rationing - basic philosophical principles and the practice; in Breyer F, Kliemt H, Thiele F (Hrsg): Rationing in Medicine: Ethical, Legal and Practical Aspects. Berlin, Springer, 2002, p 109.

16 Breyer F, Zweifel P, Kifmann, M: Gesundheitsökonomie, 4. Aufl. Berlin, Springer, 2003.

17 Alpers A, Lo B: When is CPR futile? JAMA 1995; 273:156-158.

18 Marckmann G, Siebert U: Prioritäten in der Gesundheitsversorgung: Was können wir aus dem «Oregon Health Plan» lernen? Dtsch Med Wochenschr 2002;127:1601-1604. 